\title{
Comparison of velocity and turbulence profiles obtained with a Vectrino Profiler and PIV
}

\author{
Jay Lacey ${ }^{1, *}$, Jason Duguay ${ }^{1}$, and Bruce MacVicar ${ }^{2}$ \\ ${ }^{1}$ Department of Civil Engineering, Université de Sherbrooke, Sherbrooke, QC, Canada \\ ${ }^{2}$ Department of Civil and Environmental Engineering, University of Waterloo, Waterloo, ON, Canada
}

\begin{abstract}
Laboratory experiments were carried out in a small openchannel hydraulic flume at the Universite de Sherbrooke. A PIV and an acoustic Doppler velocity profiler (Vectrino II (VII)) were used to measure high frequency velocities in profiles along the centreline of a small openchannel flume. Two background turbulence levels were tested. Comparisons were made of mean and turbulent statistics obtained with the two measurement techniques. The results show reasonable agreement between mean streamwise and lateral velocities measured with the PIV and VII near the "sweet spot" of the VII. In contrast, mean vertical velocities deviate substantially between the two measurement methods. Turbulence statistics have somewhat similar profile shapes, yet significant bias is observed between the two measurement methods.
\end{abstract}

\section{Introduction}

The Nortek Vectrino Profiler (or Vectrino II (VII)) has been commercially available since 2011. The VII is an acoustic Doppler velocity profiler (ADVP), which measures high frequency velocities in up to 35 cells over a $0.035 \mathrm{~m}$ profile. The minimum cell size is 1 $\mathrm{mm}$ along the transmitter axis, yet can be increased to $4 \mathrm{~mm}$ to average over larger volumes if desired. The cell diameter $(6 \mathrm{~mm})$ is fixed by the transmitter diameter. In principal, the new instrument represents a great advancement on currently available velocity instrumentation (especially for in situ applications). Yet preliminary studies using the VII have raised issues concerning the reliability of the measured velocities and estimated turbulence statistics [1]. Given these concerns, few studies have to date been published based on VII data. Leng and Chanson [2] conducted a thorough comparison between velocities and standard deviations obtained with the VII and a Nortek Vectrino. The authors show a noticeable disparity between the two instrument velocity measurements (as shown in authors' Figure 5). Leng and Chanson [2] conclude that the instruments showed close agreement, yet admit that the agreement is within an order of magnitude.

Given the data quality issues raised by users, in 2016 NortekAS, Norway, offered a free recalibration of user owned VIIs. Published studies investigating the reliability of the new calibration have to date not appeared in the literature. The current study investigates velocity measurements obtained using a VII that was recalibrated in $03 / 2016$. Overlapping vertical centreline profiles were obtained with the VII under 2 background turbulence

* Corresponding author: Jay.Lacey@usherbrooke.ca 
conditions (i.e., smooth, rough). Turbulence was adjusted by adding a bluff body (rectangular block) on the bed of the flume upstream of the test section. The measured velocities (and estimated turbulence statistics) are compared with velocity measurements obtained using stereo particle image velocimetry (PIV). The comparison provides new insights on the reliability of the VII and raises questions about future applications.

\section{Methods}

The experiments were performed in a $2.05 \mathrm{~m}$ long open-channel flume with a width of 0.15 $\mathrm{m}$ and a height of $0.2 \mathrm{~m}$ (Fig. 1). A flow rate of $10.15 \mathrm{l} / \mathrm{s}$, measured using a transit-time ultrasonic flow meter (Hedland), was supplied to the head tank by 2 centrifugal pumps. Flow was straitened though an aluminium honeycomb $(0.05 \mathrm{~m}$ long, $0.0075 \mathrm{~m}$ diameter $)$ before entry into the flume. The VII was positioned along the flume centreline at a lateral distance, $y=0.075 \mathrm{~m}$, approximately two-thirds downstream of the flume entrance at $x=$ $1.415 \mathrm{~m}$ (where $x$ is the longitudinal distance from the flume entrance). The field of view (FOV) for the PIV system was a vertical centreline plane approximately $\Delta x=0.14 \mathrm{~m}$ and $\Delta z=0.12 \mathrm{~m}$ which covered the entire flow depth, where $z$ is the vertical distance. The centre of the FOV was aligned to overlap the VII sampling locations.

Smooth- and rough-bed conditions were investigated. Additional turbulence was introduced to the flow by placing a $0.017 \mathrm{~m}$ thick, $0.132 \mathrm{~m}$ long, $0.098 \mathrm{~m}$ wide steel plate $0.5 \mathrm{~m}$ upstream of the VII sampling location (at $x=0.914 \mathrm{~m}$ ). During the trials, the water depth was maintained constant at $H=0.12 \mathrm{~m}$. The VII was mounted on a traversing frame and special attention was made to ensure that the probe was vertical (deviation from the vertical axis was measured as 0.14 degrees). In order to have a vertical light sheet for the PIV measurements, it was necessary to place the laser above the flume and have a transparent Plexiglas plate on the free surface just above the FOV. Given that this plate could affect the flow field, a plate of the same dimensions was used with the VII measurements. A cross was cut in the plate to allow the VII probe to descend through the centre.

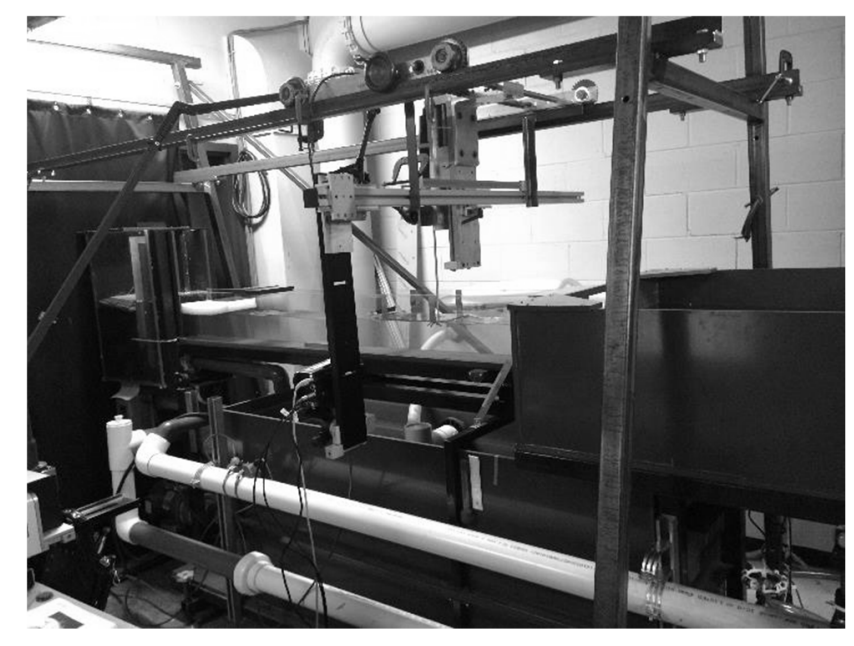

Figure 1: experimental setup.

The VII measured a $\Delta z=0.035 \mathrm{~m}$ long profile of instantaneous three-component (3C) velocities from $0.04 \mathrm{~m}$ to $0.074 \mathrm{~m}$ below the transmitter. The location of highest signal-tonoise ratio is located at $0.05 \mathrm{~m}$ from the transmitter, in a zone commonly referred to as the "sweet spot". The VII probe tip must be submerged in water, and given the blanking 
distance of $0.04 \mathrm{~m}$, the first cell down from the VII tip was located at a height of $z=0.068$ $\mathrm{m}$. Four $50 \%$ overlapping profiles were measured with the $1^{\text {st }}$ (top most) cell located at $z=$ $0.068 \mathrm{~m}, 0.051 \mathrm{~m}, 0.034 \mathrm{~m}$ and $0.017 \mathrm{~m}$. The four vertical profiles were repeated under 4 user set settings: 1) cell size $\Delta z=0.001 \mathrm{~m}$ and minimum ping interval; 2) cell size $\Delta z=$ $0.001 \mathrm{~m}$ and maximum ping interval; 3 ) cell size $\Delta z=0.002 \mathrm{~m}$ and minimum ping interval; and 4) cell size $\Delta z=0.002 \mathrm{~m}$ and maximum ping interval. The VII manual suggests using the minimum and maximum ping intervals for high and low turbulent flows, respectively [3]. The VII has as well an adaptive ping algorithm, which automatically adjusts the ping timing to minimize measurement noise, yet this setting was not used since it would potentially bias the estimated mean and turbulence statistics between compared profiles. A five minute sampling time was used for each profile collected at $50 \mathrm{~Hz}$ resulting in time series with a total of 15000 samples. To ensure sufficient seeding for the VII measurements, seeding particles were added incrementally until there was no longer a noticeable effect on signal amplitude. The VII data was treated and evaluated for quality using MITT [4]. Data was despiked using the phase-space algorithm [5] included in MITT. Using the redundant vertical velocity measuremen $\bar{w} \mathrm{t}$, the variance noise ratio was estimated by MITT (see [6]). Data with a variance noise ratio greater than $10 \%$ was identified as poor data. This relatively high standard was used to identify the "good" quality data. Data that fell outside of this range was identified and kept for comparative analysis with the "good" data. In general, only data near the "sweet spot" passed the quality control (approximately between $0.045 \mathrm{~m}$ and $0.055 \mathrm{~m}$ from the transmitter).

Stereoscopic particle image velocimetry (PIV) was carried out to provide comparative instantaneous $3 \mathrm{C}$ velocities over a plane intersecting the volume profiled by the VII. PIV measurements were taken at a rate of $400 \mathrm{~Hz}$, with two high-speed cameras (Phantom M110) fitted with $105 \mathrm{~mm}$ Nikkor lenses. The flow field was illuminated with a Litron LDY303 Nd:YLF dual cavity Q-switched laser with an output energy of $20 \mathrm{~mJ}$. The laser light sheet was spread out using a $10 \mathrm{~mm}$ concave divergent lens and thinned by a $1000 \mathrm{~mm}$ convex convergent lens. Due to restricted on-board camera RAM and the frequency floor for the laser, a five minute sampling duration was not possible. Therefore, 5 sets of 20 second continuous PIV recordings were taken for each bed condition, providing $100 \mathrm{~s}$ of total sampling time. Image acquisition and vector processing were performed within $\mathrm{DaVis}$ version 8.3.1 (LaVision Inc., USA). After stereo calibration, a self-calibration procedure was performed to reduce the average disparity vector to $0.13 \mathrm{~mm}$, effectively adjusting for any misalignments between the laser sheet and the two-level calibration plate. A decreasing 3 pass (i.e., $64 \times 64,32 \times 32,16 \times 16$ ) stereo cross-correlation was performed. A median two standard deviation vector removal and replacement filter was applied on the intermittent and final vector fields. The spatial resolution of the final vector field was 16 pixels with a $50 \%$ interrogation window overlap, providing a vector spacing of $1.22 \mathrm{~mm}$. Pixel locking was acceptably low, with the center of mass of the fractional displacement histogram being approximately $0.25 \mathrm{px}$.

The VII and PIV measurements were used to estimate mean velocities in the longitudinal, $u$, lateral, $v$, and vertical, $w$, components. Principal Reynolds shear stress $\left(-\rho \overline{u^{\prime} w^{\prime}}\right)$ and turbulent kinetic energy TKE $=0.5\left(\overline{u^{\prime} u^{\prime}}+\overline{v^{\prime} v^{\prime}}+\overline{w^{\prime} w^{\prime}}\right)$ were also estimated where $u^{\prime}$ is the (longitudinal) fluctuating velocity component and $\bar{u}$ is the timeaveraged mean velocity (i.e., $u^{\prime}=u-\bar{u}$ ).

\section{Results and discussion}

Bulk $u$ component velocity through the test section is $0.563 \mathrm{~m} / \mathrm{s}$ (estimated from the flow meter value and the water width/depth in the channel). In comparison, the double-averaged (space and time) $u$ component velocity estimated from the PIV data is $\langle\bar{u}\rangle=0.674 \mathrm{~m} / \mathrm{s}$ 
for the smooth- and rough-bed experiments, where the triangular brackets indicate spatial averaging. The higher value obtained from the PIV measurements is anticipated as the centreline velocity is maximized compared with the flow closer to the side walls. The PIV data was used to estimate the vertically averaged TKE for the smooth and rough-bed experiments, which are $<\mathrm{TKE}>=0.0017 \mathrm{~m}^{2} / \mathrm{s}^{2}$ and $<\mathrm{TKE}>=0.0029 \mathrm{~m}^{2} / \mathrm{s}^{2}$, respectively, indicating a $60 \%$ increase in background turbulence levels with the upstream block in place.

Comparison of cell size and ping intervals (not shown), reveal very similar $\bar{u}$ and TKE values near the VII's sweet spot regardless of the background turbulence levels (approximately from $0.044 \mathrm{~m}$ to $0.058 \mathrm{~m}$ from the transmitter). For several locations in this sweet spot region, data obtained using the maximum ping interval has a higher variance noise ratio, resulting in the points being identified as poorer quality. Outside of this region (from $0.066 \mathrm{~m}$ to $0.074 \mathrm{~m}$ ) the minimum ping interval resulted in marked deviations from the expected $\bar{u}$ profile. Large deviations (bias towards lower $\bar{u}$ ) within the profile due to bed reflections is seen for both maximum and minimum ping intervals, yet the location of the erroneous $\bar{u}$ values differs between ping interval settings. In general, the larger cell size appears to give more consistent, smoother profiles than the $1 \mathrm{~mm}$ cell size, yet at a cost of reduced spatial resolution. In general, the minimum ping interval gives slightly higher signal correlation values. This is more noticeable with the higher background turbulence condition. Given this higher correlation and the little difference observed in the $\bar{u}$ and TKE values (in the sweet spot region), subsequent analysis presented here was carried out on the $1 \mathrm{~mm}$ cell size and minimum ping interval data.

The vertical profiles of $\bar{u}$ obtained with PIV compare relatively well with the VII measurements in the sweet spot region where data quality is good (Fig. 2a,b). It can be seen from Figure 2a,b that the rough-bed "good" $\bar{u}$ values (filled red circles) line up well with the PIV data (red asterisk) in the sweet spot region. Outside of this region, velocities estimated with the VII (open circles) deviate greatly (biased towards lower magnitudes) from the PIV data. Differences between redundant (overlapping) VII measurements can be of the order of $100 \%$ (i.e., measurements obtained at the same physical location but from different regions within the vertical VII profiles). These results illustrate the importance of using only data derived from the VII sweet spot, which have passed the quality control criteria.

Similarly, over most of the measured depth, the $v$ component VII data, which have passed the stringent quality control criteria, correspond well with the values obtained with the PIV data (Fig. 2 c,d). In general, the background turbulence level (smooth- vs. roughbed) does not appear to affect this agreement. Near the bed, at $z<0.015 \mathrm{~m}$, there is an unexplained divergence between the two measurement methods and for the "good" data the divergence is greater for the rough-bed case. Throughout the flow depth, the VII measurements identified as poor data especially at the tail ends of individual profiles, deviate greatly from the PIV values.

Contrary to the other two velocity components, the $w$ component profiles obtained with the VII show very little agreement with the PIV results (Fig. 2e,f) under both smooth- and rough-bed conditions. PIV measurements indicate near zero $\bar{w}$ values for the smooth- and rough-bed, while the VII measurements indicate a marked non-intuitive negative trend. Furthermore, the vertically stacked VII profiles have an unrealistic saw-toothed shape. I.e., each individual profile has a negative slope and is shifted laterally such that $w$ measurements obtained at identical locations but with different profiles give markedly different results. The disparities between profiles are even more drastic when comparing the "poor" data at the tail ends of the profile. For example, for the smooth-bed case at $\mathrm{z}=0.01$ $\mathrm{m}, \bar{w}$ measured at the same physical location but from different regions within distinct VII profiles gives values of $\bar{w}=-0.005 \mathrm{~m} / \mathrm{s}$ and $0.025 \mathrm{~m} / \mathrm{s}$ (Fig. 2e). Comparing Figure 2e and 
$2 \mathrm{f}$, there is a slight indication that increased background turbulence may exaggerate the discrepancy between the two measurement methods (VII vs. PIV).
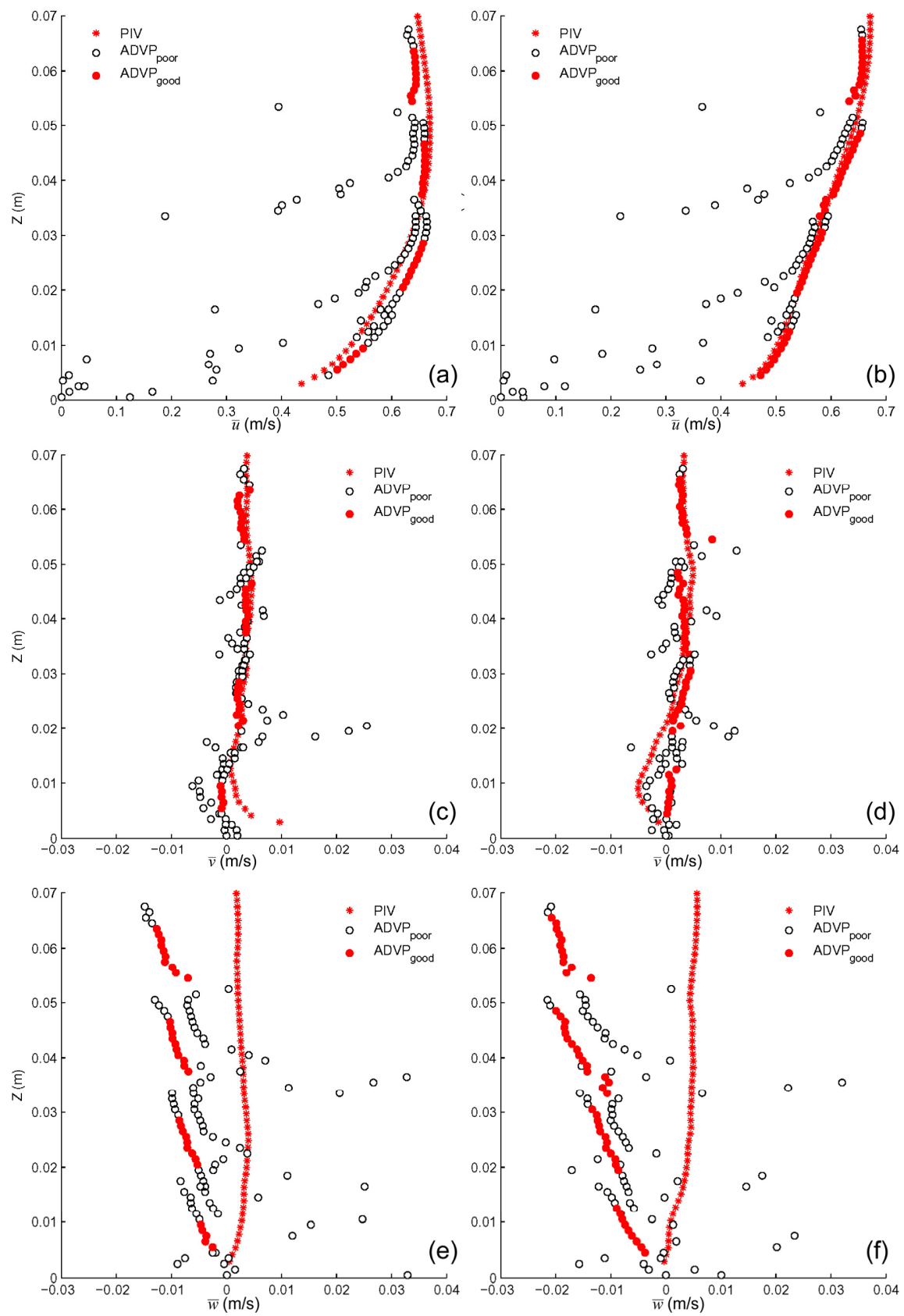

Figure 2. PIV and VII (ADVP) vertical profiles of mean longitudinal, $u$, lateral, $v$ and vertical $w$ component velocities in $\mathrm{m} / \mathrm{s}$ for the smooth-bed $(\mathrm{a}, \mathrm{c}, \mathrm{e})$ and rough-bed $(\mathrm{b}, \mathrm{d}, \mathrm{f})$ experiments. Filled circles indicate VII data, which passed quality control criteria.

TKE profiles estimated from the 3C PIV and VII velocity measurements are presented in Figure 3a,b. Both measurement techniques produce TKE profiles of somewhat similar 
shape, yet significant bias (as much as 100\%) is observed between the estimated values obtained with the 2 measurement methods. The higher TKE values estimated with the PIV are likely partially related to the smaller sampling volume and higher sampling frequency of the PIV system, which would be able to resolve finer scales of turbulence [7]. Of greater concern is that, as with the mean velocity profiles previously discussed, VII overlapping estimates of TKE can produce differences of as much as $400 \%$. Furthermore, the TKE estimated from the VII measurements for an individual profile shows a distinctive " $\mathrm{C}$ " shape with a minimum located at the sweet spot $(0.05 \mathrm{~m}$ below the transmitter). This indicates that TKE estimated by the VII is very influenced by parasitic noise, which increases non-linearly with distance from the VII's sweet spot. Given this characteristic " $C$ " shape, it is difficult to assess the actual TKE within the water column as a different value of TKE is obtained in every sampling bin. Similarly, previous research by MacVicar et al. [1] has reported "C" shaped standard deviation profiles obtained with a VII in an open-channel flow. In the region close to the sweet spot, TKE values for the smooth-bed are relatively consistent throughout the water column (Fig. 3a). In contrast, under higher background turbulence conditions, TKE values estimated from the VII in the sweet spot region very markedly (by as much as $80 \%$ ) as can be seen in Figure $3 \mathrm{~b}$.
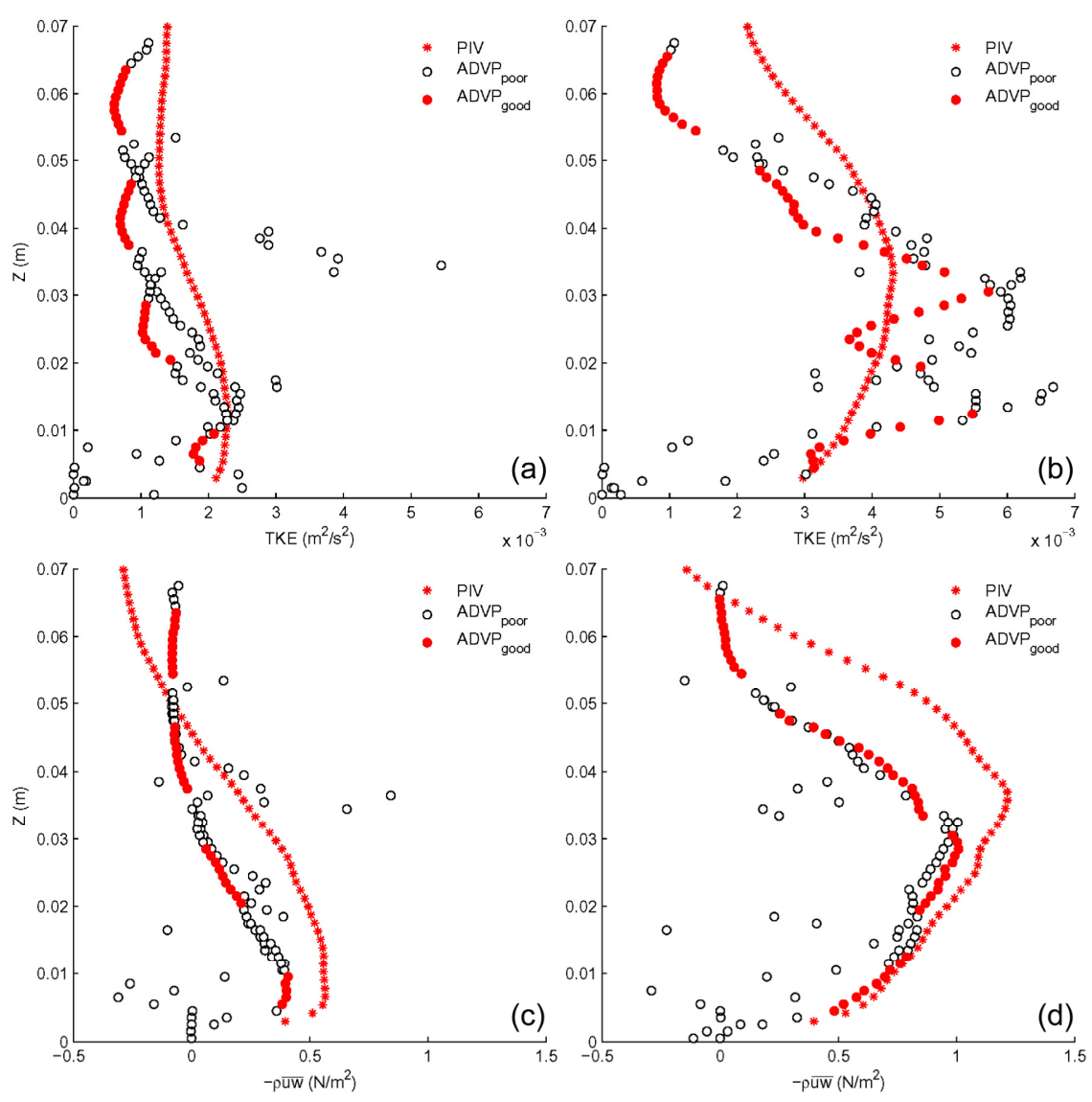

Figure 3. PIV and VII (ADVP) vertical profiles of TKE $\left(\mathrm{m}^{2} / \mathrm{s}^{2}\right)$ and $-\rho \overline{u^{\prime} w^{\prime}}\left(\mathrm{N} / \mathrm{m}^{2}\right)$ for the smoothbed $(a, c)$ and rough-bed $(b, d)$ experiments. Filled circles indicate VII data which passed quality control criteria. 
Profiles of the principal Reynolds shear stress $-\rho \overline{u^{\prime} w^{\prime}}$ estimated with the two measurement methods and bed conditions are presented in Figure $3 \mathrm{c}, \mathrm{d}$. In theory, estimates of $-\rho \overline{u^{\prime} w^{\prime}}$ should be noise free, as the parasitic white noise should be averaged out. VII vertical profiles of $-\rho \overline{u^{\prime} w^{\prime}}$ for the smooth-bed experiments do show more consistent values between overlapping profiles throughout the water column with the exception of the extreme tails of the profiles and near the bed at $\mathrm{z}<0.01 \mathrm{~m}$ (Fig. 3c). Under higher background turbulence levels, overlapping profiles are less consistent and $-\rho \overline{u^{\prime} w^{\prime}}$ values outside of the sweet spot zone very markedly. As with the TKE profiles, $-\rho \overline{u^{\prime} w^{\prime}}$ estimated from the PIV measurements show a bias $(>100 \%)$ towards higher values. In general, the bias increases with distance from the bed and is likely, in part, due to differences in sampling volume size and sampling rate as previously discussed (see [7]).

\section{Conclusions}

Our study compares mean velocities and turbulent statistics obtained with a Nortek Vectrino Profiler (VII) and a stereo PIV system. Of the statistics investigated, only mean longitudinal and lateral velocities have similar values between the two measurement techniques and these only near the "sweet spot" of the VII. In contrast, mean vertical velocities, TKE and $-\rho \overline{u^{\prime} w^{\prime}}$ deviate substantially between the two measurement methods. Furthermore, estimates of these statistics depend greatly on where they are measured with the VII profile. For example, the TKE profiles present a characteristic " $C$ " shape making it difficult to evaluate the actual TKE in the flow. Further work is necessary to properly assess the VII under various environmental conditions and at present only values at or near the instrument's sweet spot should be used for characterising flow structure.

The authors extend their gratitude to the Natural Science and Engineering Research Council of Canada for providing funding to pursue this research. We would also like to thank Nicolas Simard for his invaluable technical assistance in preparing the experiments and Dr. Eva Enders of the Department of Fisheries and Oceans, Canada, for lending us their Vectrino Profiler.

\section{References}

1. B. MacVicar, S. Dilling, J. Lacey, K. Hipel, Proc. 7th Int. Conf. Fluvial Hydraul. (River Flow), Lausanne, CH, 1951-1959 (2014)

2. X. Leng, Chanson, H. Flow Meas. Inst., 54, 136-145 (2017)

3. Nortek, MIDAS Data Acquisition Software, Software Users Guide, Boston, MA, USA, 109 pp. (2015)

4. B. MacVicar, S. Dilling, R.W.J. Lacey, Comp. Geosc., 73, 88-98. (2014b)

5. D.G. Goring, V.I. Nikora, J. Hydraul. Eng., 128, 117-126 (2002)

6. R.W.J. Lacey, C. D. Rennie, J. Hydraul. Eng., 138(1): 71-84 (2012).

7. R.L. Soulsby, J. Phys. Oceanogr. 10:208-219 (1980) 